\title{
Thermal imaging of laser powder interaction zone in Ultra-High Speed Laser Cladding process
}

by P. Koruba*, J. Reiner*, *Wroclaw Univ. of Science and Technology, Wyb.Wyspianskiego. 27, 50-370 Wroclaw, Poland, piotr.koruba@pwr.edu.pl

\begin{abstract}
Laser cladding process is a coating deposition technology, where the layer of additional material is cladded due to interaction of powder particles with laser heat source and melt pool. The monitoring of interaction between laser beam and powder became more vital in specific Ultra-High Speed Laser Cladding process, since the cladding speed requires particles to be melted before reaching the substrate. Therefore, this paper presents the preliminary study of lateral measurement of powder concentration under the cladding nozzle and its temperature distribution during the process. Moreover, the evaluation of used spectral bands for observation of laser-powder interaction was carried out.
\end{abstract}

\section{Introduction}

The laser-powder interaction is a very important phenomenon that occurs during laser cladding process (Fig 1.). It becomes much more important in case of UHSLC (Ultra-High Speed Laser Cladding) [1] or EHLA (Extreme HighSpeed Laser Material Deposition) [2], where the powder particles should be melted before reaching the melt pool on the substrate surface. There are many studies concerning modelling and simulation of powder interaction with laser during laser cladding such as development of mathematical model proposed in [3]. However, the verification of computational results regarding temperature distribution of powder heated up by laser beam is performed rarely [4]. Powder temperature measurement should be considered as one of the basic steps to obtain information about the laser cladding process, especially UHSLC process. On the other hand, geometrical measurements of powder stream distribution are made much more frequently. The basic geometrical quantities describing powder stream are constriction diameter $\left(d_{c}\right)$ and convergence angle $(\alpha)[5]$.

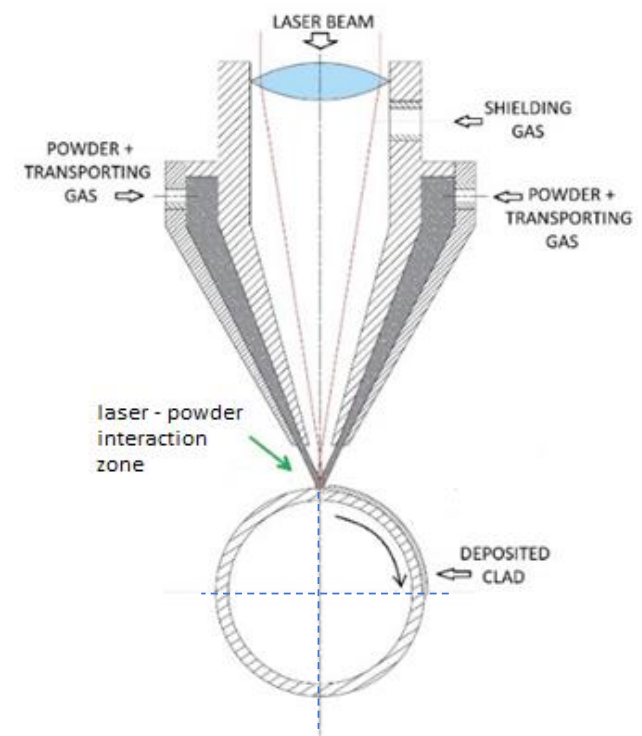

Fig. 1. Laser cladding process of cylindrical substrate with laser - powder interaction zone marked [6]

Another example of evaluation for digital simulation of powder stream concentration and its interaction with laser beam was carried out with usage of dedicated system for thermal spraying - SprayWatch, which is based on twocolor pyrometry. On the other hand, in [7] experimental assessment of performed calculations was conducted with IR pin-hole photodetector, yet this method requires additional scanning system and can be considered as time-consuming. Authors of [8] conducted the feasibility test of infrared camera application in order to visualize the powder jet in Cold Spray process, but the quantitative temperature measurement was not mentioned. Moreover, the usage of CCD camera for estimation of temperature field in powder stream during laser cladding process was described in [9], but the authors presented only image processing techniques for obtained images.

In case of NIR/SWIR spectral band, there was presented a possibility of laser welding online diagnostic [10]. The authors emphasized that the NIR camera allows visualizing and detecting the common flaws and defects within laser 
welding process as well as gives images with higher contrast than VIS camera. It is also worth mentioning that according to Plank's law the thermal radiation of molten metal powder can be easily detected by NIR/SWIR camera, whereas in case of measurement with MWIR or LWIR camera the simplified emissivity value estimated in paper [11]. However, the methodology of emissivity value is limited to powder beds, it can serve as a sufficient approximation for thermographic measurements.

This paper treats of lateral measurement of powder stream concentration and temperature distribution during the laser cladding process in order to visualize and optimize it. For this purpose, the cameras for different spectral bands were utilized from VIS to LWIR. The qualitative and quantitative comparison of the camera reading has been carried out. Furthermore, the evaluation of selected measurement spectral bands in case of laser-powder interaction visualization was performed.

\section{Experimental setup}

The experimental setup for UHSLC process consisted of manipulation system RV60-40 robot (REIS) and lathe machine Compact 300 (erba), laser unit LDF 4000-30 (Laserline), laser cladding nozzle COAXpowerline (IWS Fraunhofer) and powder feeding system H-PF2/2 (GTV). The powder material used in the process was Stellite 6 , while the shielding gas was argon. The complex experimental setup for powder stream measurements was shown on Fig. 2 , consisting of two systems for powder stream visualization and temperature measurement. These systems are going to be described separately. On the other hand, the laser cladding process parameters used during the measurement with cameras were shown in table 1.

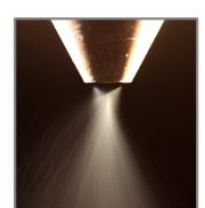

(1)
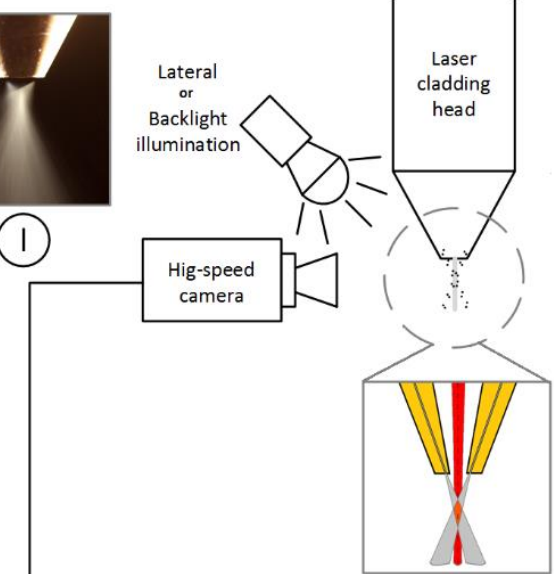

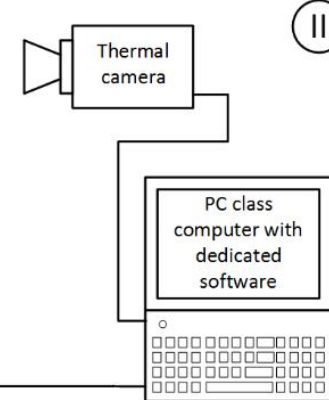

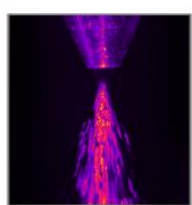

(11)

Fig. 2. Schematic of experimental setup in UHSLC process for determination of powder concentration (I) and measurement of temperature during laser-powder interaction (II)

Table 2. UHSLC process parameters used during the tests

\begin{tabular}{|c|c|c|c|c|c|c|}
\hline Parameter & Laser powder & Powder feed & Shielding gas flow & Carrying gas flow & $\begin{array}{c}\text { Laser focus } \\
\text { diameter }\end{array}$ & Rayleigh length \\
\hline Value & $500 \mathrm{~W}$ & $3.98 \mathrm{~g} / \mathrm{min}$ & $13 \mathrm{l} / \mathrm{min}$ & $4 \mathrm{l} / \mathrm{min}$ & $1.6 \mathrm{~mm}$ & $18.9 \mathrm{~mm}$ \\
\hline
\end{tabular}

\subsection{Powder concentration measurement}

For determination of the powder concentration under the cladding nozzle the experimental setup was selected as follows: Phantom v710 high speed camera, two halogen lamps and Led matrix illuminator. The usage of two different light sources was to obtain two different illumination techniques for displaying the flow of powder stream. During the measurements dark field as well as backlight illumination was used. Both of this method allow enhancing contrast of the edges of imaging object such as powder stream [13] and therefore permit quantitative characterization of powder stream from cladding nozzle. Furthermore, on the basis of illumination, the spatial distribution of the powder can be determined with the image processing (segmentation, edge detection).

\subsection{Temperature distribution measurement}

In order to carry out extended research on laser-powder interaction during UHSLC process several cameras for different spectral bands were utilized, wherein some cameras basic data was gathered in table 3 . 
Table 3. Basic properties of the used cameras

\begin{tabular}{|l|l|l|l|}
\hline Spectral band & \multicolumn{1}{|c|}{ Detector type } & Resolution & \multicolumn{1}{c|}{ Camera } \\
\hline $0.4-1.0 \mu \mathrm{m}$ & $\mathrm{Si}$ & $1292 \times 964$ & Allied Vision Mako G-125 \\
\hline $0.9-1.7 \mu \mathrm{m}$ & $\mathrm{InGaAs}$ & $320 \times 256$ & Allied Vision Goldeye G-008 \\
\hline $1.5-5.1 \mu \mathrm{m}$ & InSb & $320 \times 256$ & FLIR SC7500MB \\
\hline $8.0-14 \mu \mathrm{m}$ & VOx microbolometer & $320 \times 240$ & NEC Avio TVS200 \\
\hline
\end{tabular}

It is worth noticing that due to danger of damaging the matrix by scattered laser radiation( $910 \mathrm{~nm}, 940 \mathrm{~nm}, 980$ $\mathrm{nm}, 1030 \mathrm{~nm}$ ) a long-pass filter FEL1150 with cut-on wavelength $1150 \mathrm{~nm}$ was mounted in optical system of Goldeye G008 SWIR camera. Furthermore, in case of SC7500 MWIR camera, a narrow band sapphire filter CWL $3.99 \mu \mathrm{m}$ was mounted on rotating filter wheel in order to extent the temperature measuring range. In standard configuration this thermal camera allows using multirange measurement within $5 \div 300{ }^{\circ} \mathrm{C}$ temperature range, whereas the usage of sapphire filter permits the measurement in the range from $300{ }^{\circ} \mathrm{C}$ to $1500{ }^{\circ} \mathrm{C}$. In summary, it gives six spectral bands that were used in this study for laser-powder interaction observation and powder temperature distribution visualization:

- $\quad 0.4-1.0 \mu \mathrm{m}$ with VIS camera Mako G-125 (II-a)

- $\quad 0.9-1.7 \mu \mathrm{m}$ with SWIR camera Goldeye G-008 (II-b)

- $\quad 1.15-1.7 \mu \mathrm{m}$ with SWIR camera and FEL1150 filter (II-c)

- $\quad 1.5-5.1 \mu \mathrm{m}$ with MWIR camera SC7500 (II-d)

- $\quad 3.97-4.01 \mu \mathrm{m}$ with MWIR camera and CWL 3.99 filter (II-e)

- $\quad 8.0-14.0 \mu \mathrm{m}$ with LWIR camera Avio TVS-200 (II-f)

In the case of cameras calibrated for temperature measurement such as FLIR SC7500 and Avio TVS-200 the powder emissivity value was set to 0.8 according to results obtained for iron powder in the research described in [12], whereas for Goldeye G-008 camera a calibration was carried out.

\section{Results and discussion}

The experiments of powder stream measurement were divided into two types. The first group concerned the characterization of powder stream without laser radiation in order to determine its geometrical properties. Therefore, a comparison of used illumination method was made. In case of laser-powder interaction visualization all the images received from cameras described above were compared and evaluated.

\subsection{Determination of powder stream concentration}

Exemplary images obtained with high speed camera are presented on Fig. 3, wherein they show the results of two different illumination methods: dark field (I-a) and backlight (I-b). It is worth emphasizing, that the both method can be used only with additional image processing such as maximum intensity search in case of $\mathrm{I}-\mathrm{a}$ and contrast enhancement for I-b. The distances from cladding nozzle determined from images are respectively $h_{1}-6.9 \mathrm{~mm}$ and $h_{2}-$ $6,0 \mathrm{~mm}$. It should be emphasized that in case of powder stream backlight method seems to be less effective and therefore dark field illumination was considered more appropriate for diagnostic of powder stream from cladding nozzle.

a)

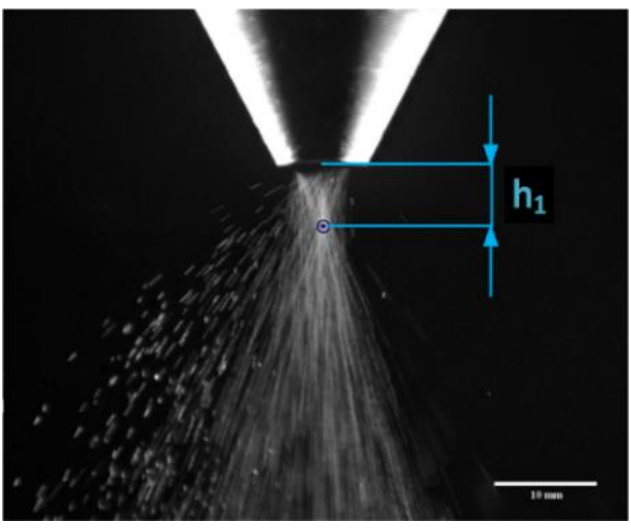

b)

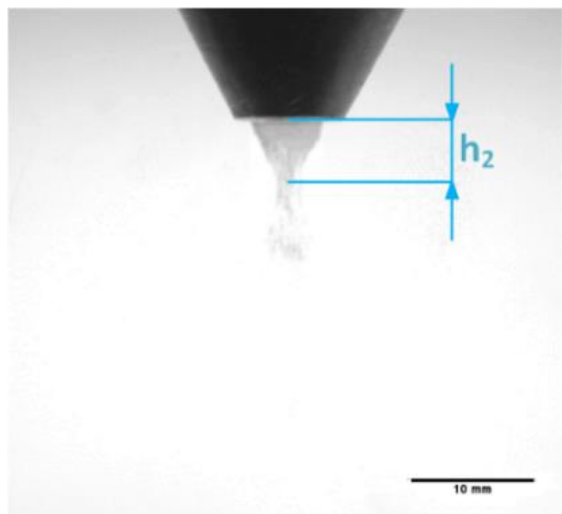

Fig. 3. Images obtained by high speed camera Phantom v710 with different illumination methods: (a) lateral halogen light dark field (I-a), (b) LED backlight (I-b) 


\subsection{Determination of powder temperature distribution - cameras comparison}

In this section the comparison of images obtained with different cameras and various spectral bands was conducted. Firstly, on Fig. 4 the intensity image received in VIS range received by Mako G-125 camera was presented. It clearly shows the scattering of laser beam on powder particles and therefore this method might be competitive to the dark field illumination method described earlier.

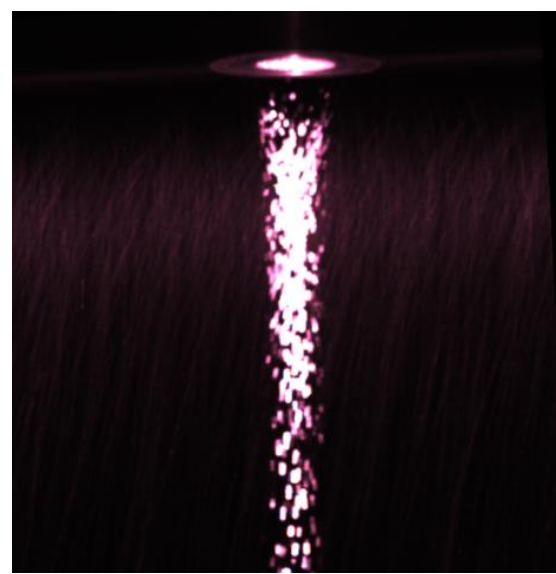

Fig. 4. Image of laser beam scattering on powder particles obtained with VIS/NIR Mako G-125 camera (II-a)

The resultative images obtained with SWIR camera were shown on figure 6 . In case of laser-powder interaction zone image acquisition without long-pass filter (Fig $5 a$ ), it can be stated that the laser scattering is significantly visible and the zone, where laser beam the most affect the powder particles can be determined. One can also observe beam attenuation along the powder stream. However, observation of laser beam interaction with powder without long-pass filter should be considered hazardous in case of SWIR camera matrix damage. On the other hand, figure $5 \mathrm{~b}$ shows the image with the filter in optical system of the camera and now one can observe only the heating of the powder particles. In order to conduct quantitative analysis of temperature distribution a calibration for SWIR Goldeye G-008 camera was proposed. It is described in more detail later in the text.

a)

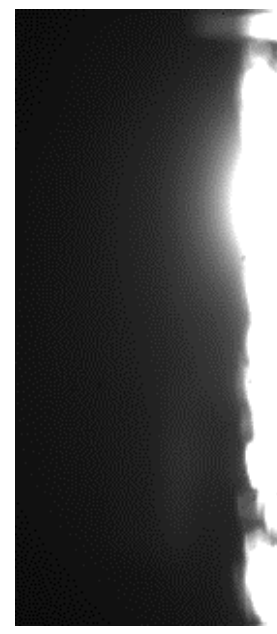

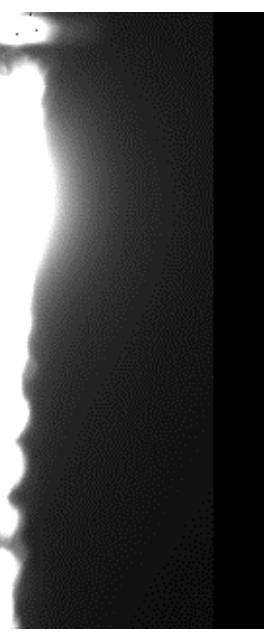

b)

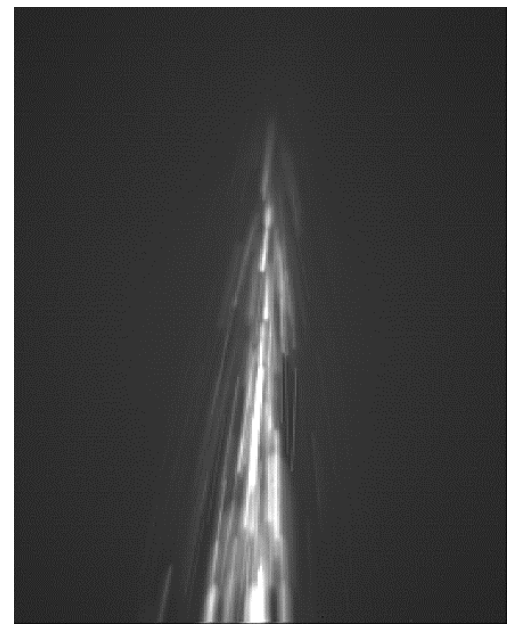

Fig. 5. Intensity images of laser - powder interaction obtained with SWIR Goldeye G-008 camera: (a) without long-pass FEL1150 filter (II-b), (b) with long-pass FEL1150 filter (II-c)

The next measurement of powder temperature distribution was performed in MWIR range with FLIR SC7500 camera, using multi IT (integration time) option available in camera for more precise temperature measurement. With the usage of standard temperature range for this infrared camera a thermal image shown on figure 6a was obtained. It is very important that in this case the maximum of the range is $300{ }^{\circ} \mathrm{C}$, what may be considered as too low temperature during laser - powder interaction, since the powder particles can start melting at that time. The more intriguing is an observation of low temperature values when using narrow band filter CWL $3.99 \mu \mathrm{m}$ (Fig. 6b), but this could be explained 
by the shielding gas (argon) infrared spectra transmission presented in [14]. Nevertheless, it should be emphasized that shielding gas transmission becomes a key issue in case of temperature measurement in IR spectrum.

a)

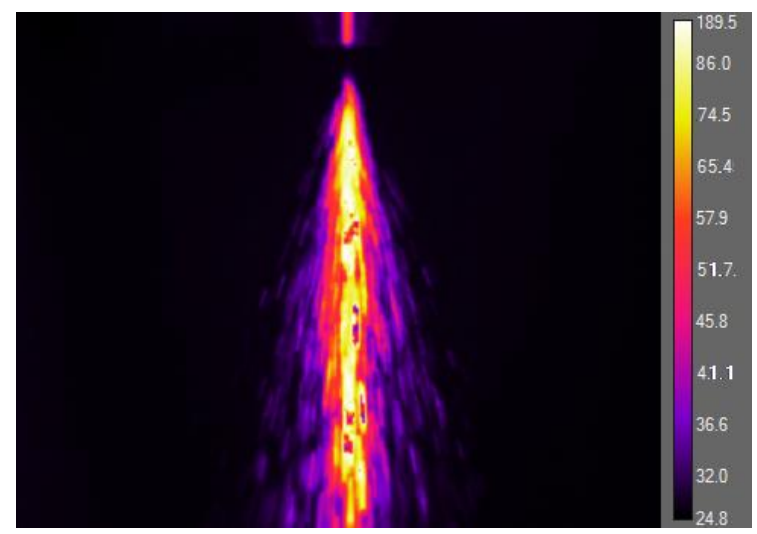

b)

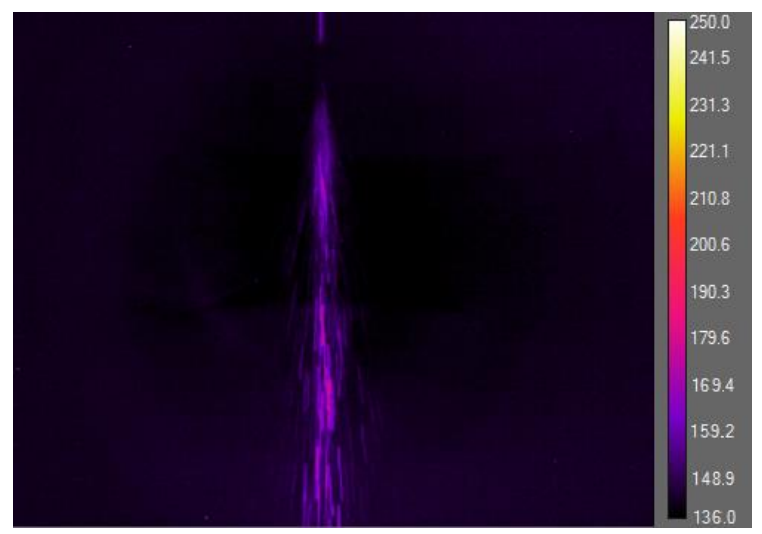

Fig. 6. Thermal image of laser - powder interaction obtained with FLIR SC7500 camera: (a) without narrow band filter CWL $3.99 \mu \mathrm{m}$ (II-d), (b) with narrow band filter CWL $3.99 \mu \mathrm{m}$ (II-e)

The more in depth analysis was performed by comparison of maximum temperature inside the box region of interest (ROI) shown of figure $7 \mathrm{a}$ and $7 \mathrm{~b}$. The time of observation was set to 10 seconds, with frame rate $25 \mathrm{~Hz}$. The results depicted on figure 7c show that the mean value of maximal temperature during the observation is almost the same in two measurement ranges and equals about $190^{\circ} \mathrm{C}$. Furthermore, the values exceed $300^{\circ} \mathrm{C}$ only in case of standard range. This might be caused by low infrared radiation transmission by argon around the wavelength $3.99 \mu \mathrm{m}$. It is worth stressing that using standard temperature range for SC7500 camera, which more appropriate for this measured temperature levels, the standard deviation is clearly greater. This may be caused because of higher precision in this range, since with narrow band filter all of the readings has small signal to noise ration and can be almost considered as noise.

a)

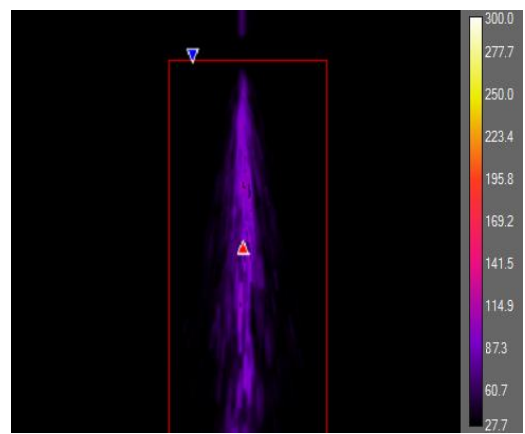

b)

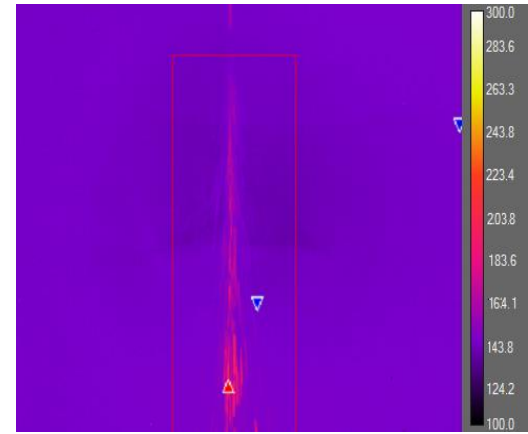

c)

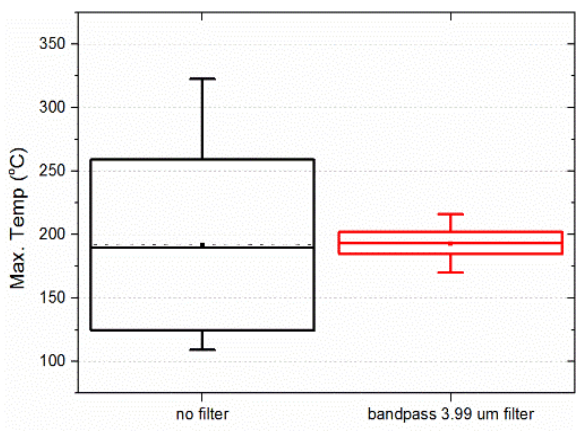

Fig. 7. Measurement of maximum temperature inside box ROI: (a) observation without narrow band filter (II-d), (b) observation with narrow band filter (II-e), (c) box chart data representation

The last observation was made using LWIR camera with similar temperature range as MWIR camera. However, in this case even lower readings were observed (Fig. 8). The maximal temperature registered during the observations fluctuated on the level of $50 \div 55^{\circ} \mathrm{C}$. It may also be caused by absorption of thermal radiation by shielding gas, since the temperature of powder particles cannot be that low during laser cladding process. This resulted in no further development of this thermal imaging system in case of online UHSLC process diagnostics.

From all the imaging systems described above the SWIR camera was chosen for further investigations. The aim of the extended study was to compare temperature distributions obtained with LWIR and MWIR camera with calculated based on data obtained with SWIR camera. Moreover the impact of process parameters on temperature measurement was taken into account, since from preliminary experiments the issue related to absorption of IR radiation by shielding gas (argon) was raised. 


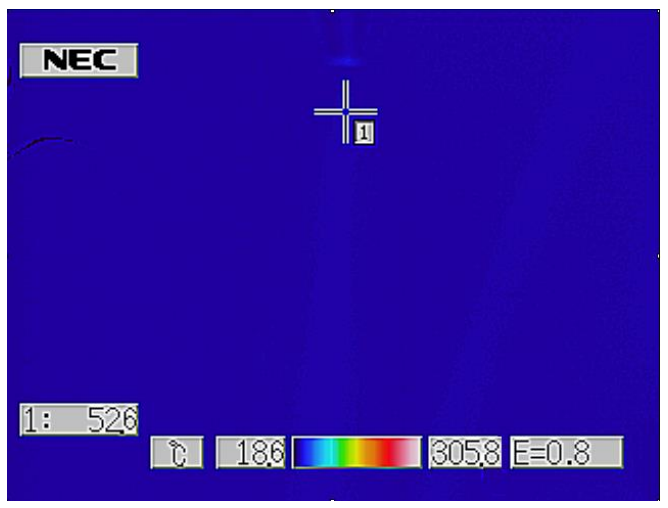

Fig. 8. Thermal image of laser - powder interaction obtained with NEC Avio TVS-200 infrared camera

\subsection{Calibration of SWIR camera for temperature measurement}

In order to analyse temperature distribution of powder particles during interaction with laser beam with images obtained with SWIR camera Goldeye G-008 a calibration procedure was performed to convert the intensity units into temperature values. As a heat source served blackbody calibrator Omega BB-4A allowing to use temperature range from $100^{\circ} \mathrm{C}$ to $982^{\circ} \mathrm{C}$. The variables during calibration were exposure time $(E T)$, f-number of the objective (f/\#) and temperature of the cavity $\left(T_{c}\right)$. The exemplary images for selected variable parameters were shown on figure 9 . All of the parameters sets used during calibration were presented in table 4.

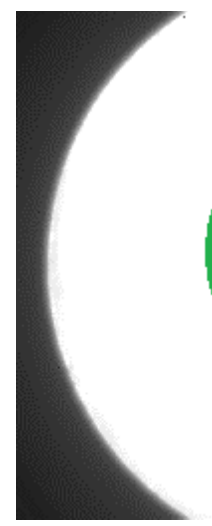

a)

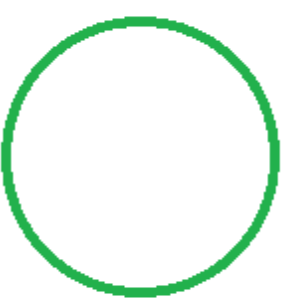

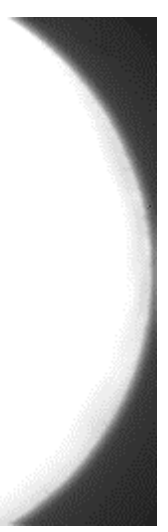

b)

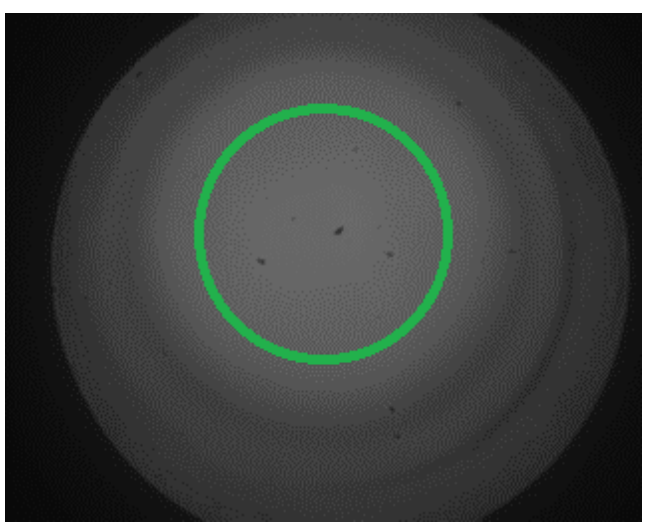

Fig. 9. Intensity images of heated to $600^{\circ} \mathrm{C}$ cavity of blackbody calibrator Omega BB-4A received with SWIR camera: (a) ET $-0.5 \mathrm{~ms}$ and $\mathrm{f} / 1.4$, (b) ET $-0.5 \mathrm{~ms}$ and $\mathrm{f} / 8$

Table 4. The scope of the temperature calibration of the SWIR camera

\begin{tabular}{|c|c|c|c|c|c|c|c|c|c|c|c|c|c|c|c|}
\hline & & \multicolumn{14}{|c|}{ Temperature $\left({ }^{\circ} \mathrm{C}\right)$} \\
\hline & & \multicolumn{2}{|c|}{300} & \multicolumn{2}{|c|}{400} & \multicolumn{2}{|c|}{500} & \multicolumn{2}{|c|}{600} & \multicolumn{2}{|c|}{700} & \multicolumn{2}{|c|}{800} & \multicolumn{2}{|c|}{900} \\
\hline \multirow{7}{*}{ 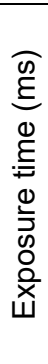 } & 0.1 & $f / 1.4$ & $f / 8$ & $f / 1.4$ & $f / 8$ & $f / 1.4$ & $f / 8$ & $\mathrm{f} / 1.4$ & $f / 8$ & $f / 1.4$ & $f / 8$ & $\mathrm{f} / 1.4$ & $f / 8$ & $\mathrm{f} / 1.4$ & $f / 8$ \\
\hline & 0.2 & $f / 1.4$ & $f / 8$ & $f / 1.4$ & $f / 8$ & $f / 1.4$ & $f / 8$ & $f / 1.4$ & $f / 8$ & $f / 1.4$ & $f / 8$ & $f / 1.4$ & $f / 8$ & $\mathrm{f} / 1.4$ & $f / 8$ \\
\hline & 0.5 & $f / 1.4$ & $f / 8$ & $f / 1.4$ & $f / 8$ & $f / 1.4$ & $f / 8$ & $\mathrm{f} / 1.4$ & $f / 8$ & $f / 1.4$ & $f / 8$ & $\mathrm{f} / 1.4$ & $f / 8$ & $\mathrm{f} / 1.4$ & $f / 8$ \\
\hline & 1.0 & $f / 1.4$ & $\mathrm{f} / 8$ & $f / 1.4$ & $f / 8$ & $f / 1.4$ & $f / 8$ & $f / 1.4$ & $f / 8$ & $\mathrm{f} / 1.4$ & $f / 8$ & $\mathrm{f} / 1.4$ & $f / 8$ & $\mathrm{f} / 1.4$ & $\mathrm{f} / 8$ \\
\hline & 2.0 & $f / 1.4$ & $f / 8$ & $f / 1.4$ & $f / 8$ & $f / 1.4$ & $f / 8$ & $f / 1.4$ & $f / 8$ & $f / 1.4$ & $f / 8$ & $f / 1.4$ & $f / 8$ & $\mathrm{f} / 1.4$ & $f / 8$ \\
\hline & 5.0 & $f / 1.4$ & $f / 8$ & $f / 1.4$ & $f / 8$ & $f / 1.4$ & $\mathrm{f} / 8$ & $f / 1.4$ & $f / 8$ & $\mathrm{f} / 1.4$ & $f / 8$ & $\mathrm{f} / 1.4$ & $f / 8$ & $\mathrm{f} / 1.4$ & $f / 8$ \\
\hline & 10.0 & $f / 1.4$ & $\mathrm{f} / 8$ & $\mathrm{f} / 1.4$ & $\mathrm{f} / 8$ & $\mathrm{f} / 1.4$ & $f / 8$ & $f / 1.4$ & $\mathrm{f} / 8$ & $\mathrm{f} / 1.4$ & $f / 8$ & $\mathrm{f} / 1.4$ & $\mathrm{f} / 8$ & $\mathrm{f} / 1.4$ & $\mathrm{f} / 8$ \\
\hline
\end{tabular}


Figure 10a shows an exemplary calibration curve that served for temperature calculation during observations of laser - powder interaction, whereas figure 10b shows an example of SWIR image with calculated greyscale bar of temperature values. In the case of SWIR camera the measured maximal temperature of powder particles was $490^{\circ} \mathrm{C}$, so it was considerably higher than with usage of MWIR camera. Moreover, it should be stressed that the calibration method used in this study might cause reduction of this value.

a)

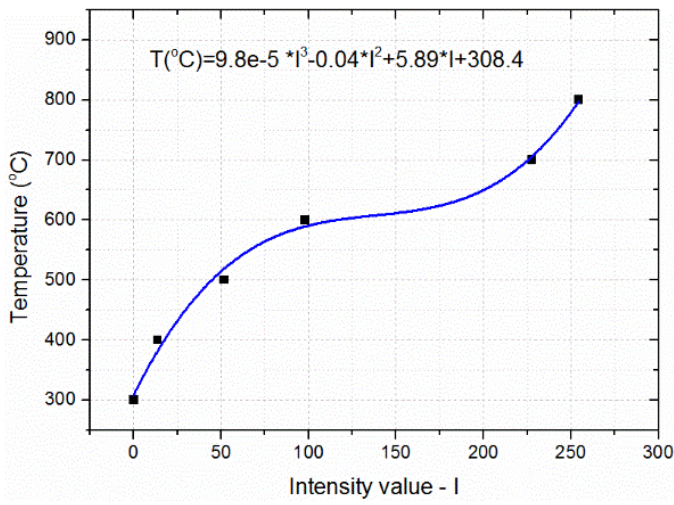

b)

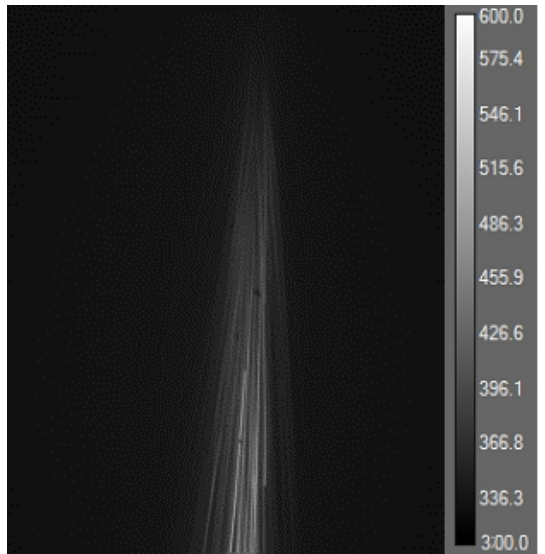

Fig. 10. Result of SWIR Goldeye G-008 camera calibration: (a) calibration curve for ET - $0.5 \mathrm{~ms}$ and f/8, (b) calibrated image for $E T-5 \mathrm{~ms}$ and $\mathrm{f} / 8$

\subsection{Impact of shielding gas on temperature measurement}

As a final verification of UHSLC process monitoring with IR camera, an impact of process parameter influencing temperature measurement was checked. As it was mentioned before argon as a shielding gas can considerably absorb infrared radiation of selected spectral bands as well as it flow may distort the results. The test relied on realization of laser cladding process without shielding gas in order to observe changes in temperature measurement. In this study MWIR SC7500 and SWIR Goldeye G-008 cameras were used.

a)

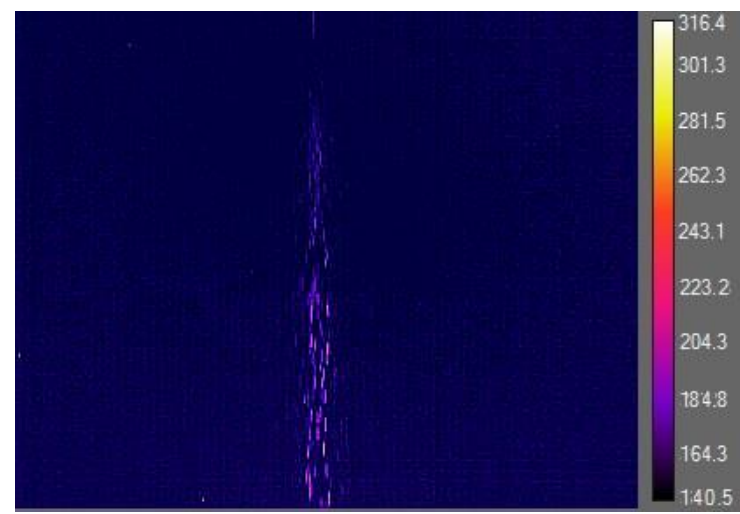

b)

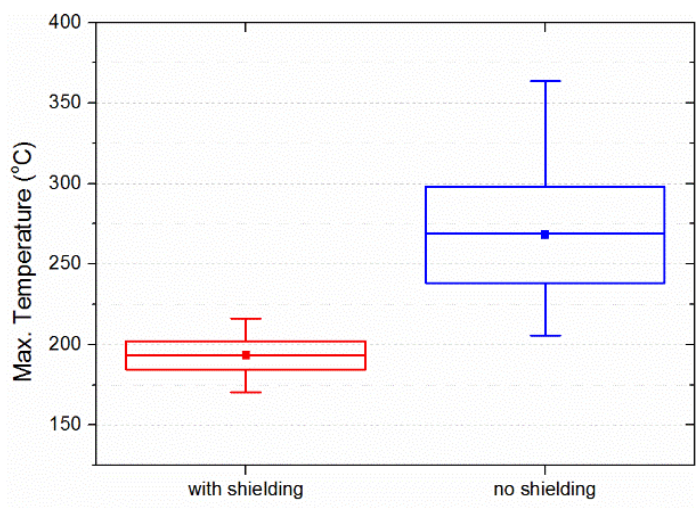

Fig. 11. Measurement of maximum temperature inside ROI: (a) observation with narrow band filter (II-d), (b) box chart data representation

The results obtained with MWIR camera (Fig. 11a) were compared with earlier discussed temperature measurements, when the shielding gas from the nozzle was activated. On figure 11b one can show the difference between this two cases, since without shielding gas the measured maximal temperature inside ROI was higher. However, it was still lower than the values received with SWIR camera because of carrying gas presence in the process. One can make a comparison based on figure 12 that shows the images obtained with SWIR camera. It is clearly visible that the temperature of powder particles is higher when shielding gas is not active. Moreover, it exceeds $600^{\circ} \mathrm{C}$, that is the maximal value of temperature for performed calibration. Therefore, a further study to choose optimal values of SWIR imaging system parameters is required. 
a)

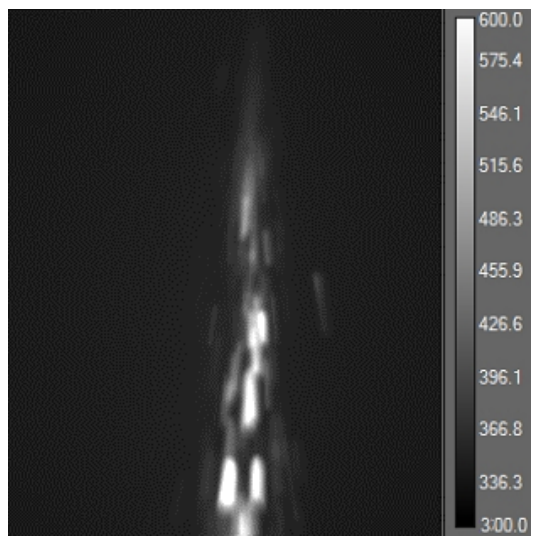

b)

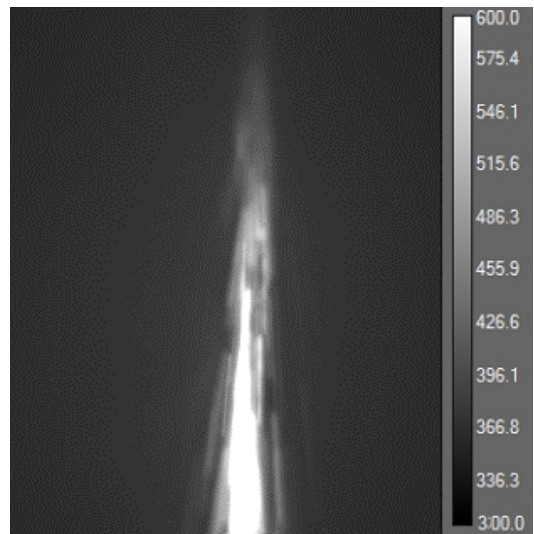

Fig. 12. Comparison of images obtained with SWIR camera for ET - $0.2 \mathrm{~ms}$ and f/1.4: (a) with shielding gas, (b) without shielding gas

\section{Conclusions}

The presented study indicates that the most adequate IR spectral band for monitoring of laser - powder interaction in UHSLC process is SWIR or MWIR band. Although VIS/NIR camera provides high resolution pictures with visible laser scattering, it mostly gives information about laser beam scattering and this radiation might be hazardous for camera matrix. In case of LWIR band the attenuation of thermal radiation by shielding gas is significant enough that such camera cannot be used for process diagnostics.

After performing temperature calibration using blackbody model the images obtained with SWIR camera were compared with MWIR images. It is worth noticing that in case of SWIR the measured temperatures were considerably higher, even though the measurement range in case of MWIR camera was larger. On the other hand, the impact of shielding gas was determined. In case of both spectral bands it reduces infrared radiation that reach the detector and this results in lower temperature reading.

\section{REFERENCES}

[1] Schopphoven T., Gasser A., Backes G., EHLA: Extreme High-Speed Laser Material Deposition, Economical and effective protection against corrosion and wear, Las. Tech. J. 2017:14(4):26-29.

[2] Koruba P., Jurewicz P., Reiner J. et al., Ultra-High Speed Laser Cladding (UHSLC) technology for Stellite 6 functional coatings deposition in aviation industry, Przeglad Spawalnictwa 2017:89(6):15-19.

[3] Fu Y., Loredo A., Martin B., et al., A theoretical model for laser and powder particles interaction during laser cladding, J. Mat. Proc. Tech. 2002:128:106-112.

[4] Wen S., Shin Y., Murthy J., Sojka P., Modelling of coaxial powder flow for the laser direct deposition process, Int. J. Heat Mass Transfer 2009:52(25-26):5867-5877.

[5] Zhirnov IV, Doubenskaia MA, Smurov IY, Teleshevskiy VI, Measurement of Basic Geometric Characteristics of Particle Flow Between Surfacing Head and Substrate, Measurement Techniques, 2017:59:11:1187-90.

[6] Radkiewicz P., Koruba P., Reiner J., Development of auxiliary gas protection during laser cladding on the axisymmetric Ti6Al4V component, Proceedings of the 14th International Scientific Conference: Computer Aided Engineering.

[7] Lin J., Temperature analysis of the powder streams in coaxial laser cladding, Opt.\& Las. Tech. 1999:31:565-570.

[8] Sova A., Doubenskaia MA, Petrovskiy P., Smurov I., Visualization of particle jet in cold spray by infrared camera: feasibility test, The Int J Adv Manuf Tech, 2018:95(5-8);3057-63.

[9] 8Li L., Yang XC, Digital Image Processing of CCD Measuring Temperature Field in Coaxial Powder Stream of Laser Cladding, Key Eng. Mat. 2009:392-394:1037-1041.

[10] Dorsch F., Braun H., Kessler S., Pfitzner D., Rominger V., Online NIR diagnostic of laser welding processes and its potential for quality assuring sensor systems, Proc. SPIE 8963, High-Power Laser Materials Processing: Lasers, Beam Delivery, Diagnostics, and Applications III, 89630R, 2014.

[11] Sih SS, Barlow JW, The Prediction of the Emissivity and Thermal Conductivity of Powder Beds, Particulate Science and Technology: An International Journal, 2004:22(3):291-304.

[12] Sih SS, Barlow JW, Emissivity of Powder Beds, in Solid freeform fabrication Proceedings, 1995: 402-408.

[13] Reiner J., Rendering for machine vision prototyping, in Proceedings of SPIE - The International Society for Optical Engineering, 2008:7100: 710009

[14] Tourin RH, Henry PM, Liang ET, Infrared Spectra of Nitrogen, Argon, and Helium Plasmajets, Journal of the Optical Society of America, 1961:51(7):800-801. 